BMJ Open Sport \& Exercise Medicine

\title{
Surgical plantaris tendon removal for patients with plantaris tendon-related pain only and a normal Achilles tendon: a case series
}

\author{
Håkan Alfredson, ${ }^{1,2,3}$ Lorenzo Masci, ${ }^{3}$ Christoph Spang ${ }^{4}$
}

\begin{abstract}
To cite: Alfredson H, Masci L, Spang C. Surgical plantaris tendon removal for patients with plantaris tendon-related pain only and a normal Achilles tendon: a case series. BMJ Open Sport \& Exercise Medicine 2018:4:e000462. doi:10.1136/ bmjsem-2018-000462
\end{abstract}

Accepted 12 November 2018

Check for updates

(C) Author(s) (or their employer(s)) 2018. Re-use permitted under CC BY-NC. No commercial re-use. See rights and permissions. Published by BMJ.

${ }^{1}$ Sports Medicine Unit, Department of Community Medicine and Rehabilitation, Umeå University, Umeå, Sweden ${ }^{2}$ ISEH, UCLH, London, UK ${ }^{3}$ Pure Sports Medicine Clinic, London, UK

${ }^{4}$ Anatomy Section, Department of Integrative Medical Biology, Umea University, Umeå, Sweden

Correspondence to Mr Christoph Spang; Christoph. Spang@umu.se

\section{ABSTRACT}

Objectives Surgical removal of the plantaris tendon can cure plantaris-associated Achilles tendinopathy, a condition in which Achilles and plantaris tendinopathy coexist. However, rare cases with plantaris tendinopathy alone are often misdiagnosed due to a normal Achilles tendon. Design and setting Prospective case series study at one centre.

Participants Ten consecutive patients ( 9 men and one woman, mean age 35 years, range 19-67) with plantaris tendon-related pain alone in altogether 13 tendons were included. All had had a long duration (median 10 months, range 3 months to 10 years) of pain symptoms on the medial side of the Achilles tendon mid-portion. Preoperative ultrasound showed thickened plantaris tendon but a normal Achilles tendon.

Interventions Operative treatment consisting of ultrasound-guided excision of the plantaris tendon. Primary and secondary outcome measures Scores from Victorian Institute of Sports Assessment-Achilles questionnaire (VISA-A)were taken preoperatively and postoperatively (median duration 10 months). Patient satisfaction and time until full return to sports activity level was asked by a questionnaire.

Results The VISA-A scores increased from 61 (range 4581) preoperatively to 97 (range 94-100) postoperatively $(p<0.01)$. Follow-up results at 10 months (range $7-72$ months) on $9 / 10$ patients showed full satisfaction and return to their preinjury sports or recreational activity Conclusion The plantaris tendon should be kept in mind when evaluating painful conditions in the Achilles tendon region, especially when no Achilles tendinopathy is present. Excision of the plantaris tendon via a minor surgical procedure in local anaesthesia results in a good outcome.

\section{INTRODUCTION}

Chronic pain in the Achilles tendon mid-portion is a quite common condition among professional and recreational athletes. Treatment can often be very challenging especially during the season. ${ }^{1}$ Despite good clinical outcomes of conservative measures such as heavy load eccentric training, ${ }^{2}$ several
What are the new findings?

- Plantaris tendinopathy can occur without Achilles tendinopathy.

- Plantaris tendinopathy may be misdiagnosed because ultrasound and MRI examination show a normal Achilles tendon.

- Excision of the plantaris tendon in patients with plantaris tendon-related pain (plantaris tendinopathy) results in pain relief and fast return to full sports activity.

patients still need to be operated for a proper pain relief. ${ }^{3}$

In many of those non-responding cases a coexisting tendinopathy in the neighbouring plantaris tendon has been found, ${ }^{45}$ especially in those where the pain is located rather medially. ${ }^{6}$ Surgical explorations have detected a thickened plantaris tendon in close vicinity to the medial Achilles tendon ${ }^{7}$ leading to interference between the two tendons during certain movements. ${ }^{8}$ Anatomical studies have found up to nine different insertional areas of the plantaris tendon among the population, ${ }^{9}{ }^{10}$ some potentially influencing the Achilles tendon. ${ }^{11}{ }^{12}$ Surgical removal of the plantaris tendon in patients with plantaris-associated mid-portion Achilles tendinopathy has resulted in very good clinical outcomes ${ }^{13-16}$ including structural improvement of the medial Achilles tendon area. $^{1516}$

New clinical experiences show that in a few cases plantaris tendinopathy alone, without coexisting mid-portion Achilles tendinopathy, occurs and causes debilitating pain in the Achilles tendon region. These patients are often misdiagnosed because ultrasound (US) and MRI examinations show a normal Achilles tendon. This study aimed to describe typical clinical and US findings in this rare condition, and also to prospectively follow 

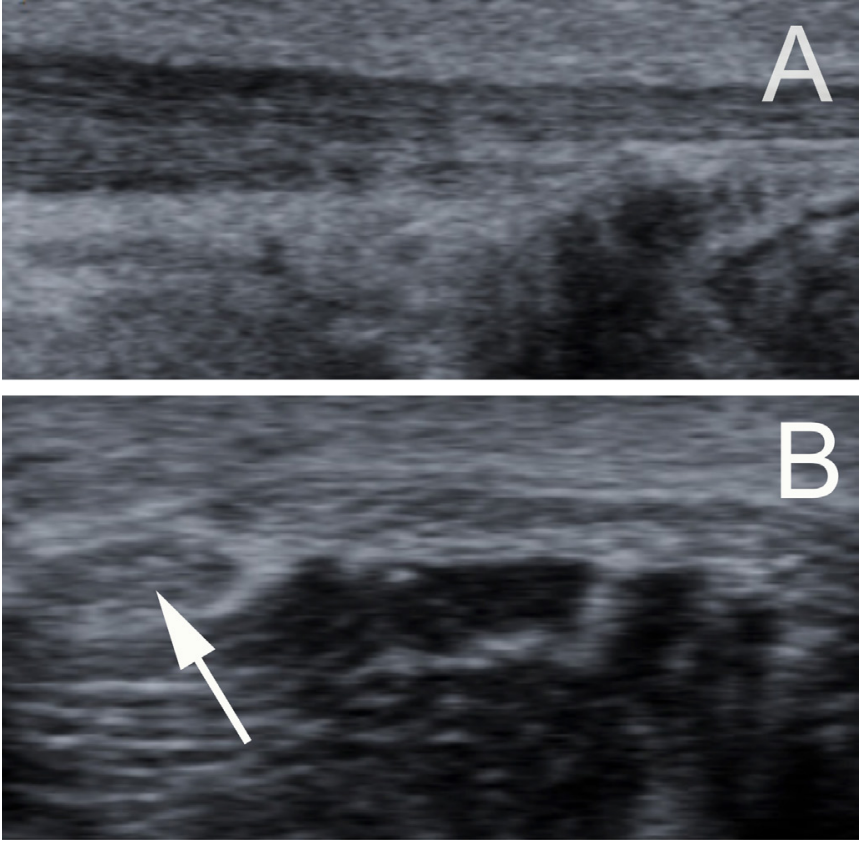

Figure 1 Ultrasound examination of a patient with a normal Achilles tendon (longitudinal view) (A). Cross view showing a thickened plantaris tendon (arrow) on the medial/ventral side of the Achilles (B).

the clinical results after surgical treatment with plantaris tendon removal.

\section{METHODS}

\section{Patients}

The study includes a case series of 10 consecutive patients (9 men and one woman, mean age 35 years, range 19-67) with plantaris tendon-related pain alone in altogether 13 tendons. All patients were sports active, ranging from national high level in sprint, hurdles, long jump and handball to recreational jogging. All had had a long duration (median 15 months, range 3 months to 120 months) of pain symptoms on the medial side of the Achilles tendon mid-portion. Typical symptoms and history include occasions with sharp pain on the medial side of the Achilles tendon mid-portion during explosive ankle joint movements like sprinting. Symptoms often subsided quickly within 3-4 days, and the traditional eccentric exercises used for treatment of mid-portion Achilles tendinopathy often caused a worsening with more pain on the medial side.

\section{Presurgical examinations \\ Clinical examination}

Clinically all patients showed a non-thickened and non-tender Achilles tendon mid-portion. Local tenderness was only found medially to the Achilles tendon mid-portion.

\section{US+colour Doppler examination}

Examination via US+colour Doppler showed a normal Achilles tendon (figure 1A). No thickening, no hypoechogenicity and no high blood flow were detected.

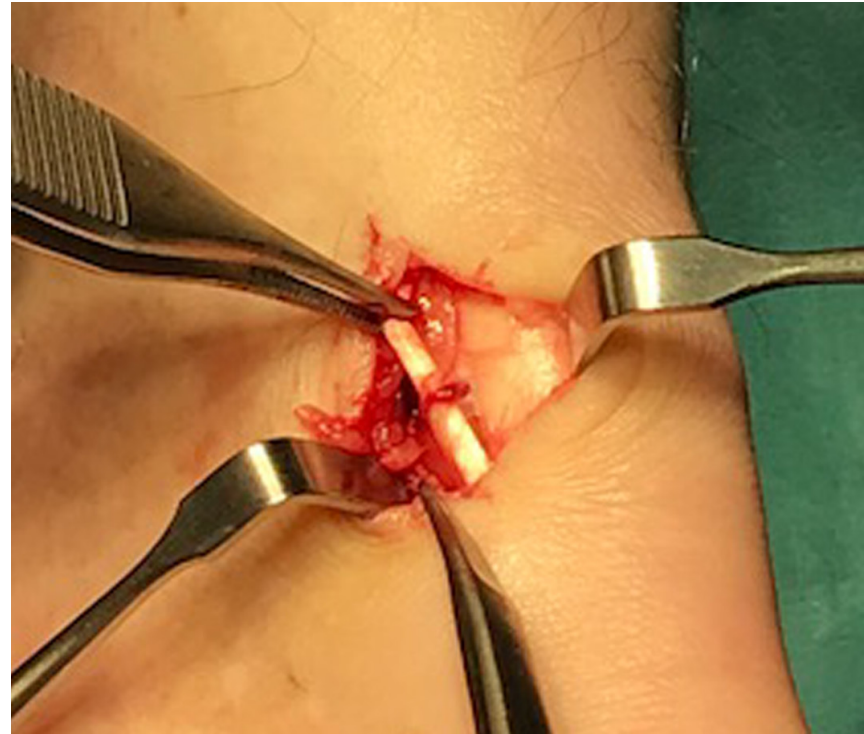

Figure 2 Surgical exploration in local anaesthesia via a $1 \mathrm{~cm}$ long skin incision on the medial side of the Achilles tendon mid-portion in a patient with plantaris tendinopathy. The plantaris tendon is seen embedded in vascularised fat tissue on the medial side of the Achilles (A). Extirpation of 5-6 cm of the plantaris tendon and adjacent fat tissue .

Dynamic US examination following the plantaris from proximal to distal showed a thickened plantaris tendon (arrow) located close to the medial side of the Achilles tendon mid-portion (figure 1B). ${ }^{6}$

\section{Surgical procedure}

During surgical treatment under local anaesthesia, the medial aspect of the Achilles tendon was visualised and carefully inspected (figure 2). The exact location and macroscopic appearance of the plantaris tendon was identified. The surgical treatment consisted of a release of the plantaris tendon followed by excision distally from the calcaneal insertion and proximally at a level slightly above the distal medial soleus muscle insertion. In contrast to the previously described procedure there was no need for treatment of the medial aspect of the (normal) Achilles tendon. ${ }^{67}$

\section{Outcome measures}

For evaluation before surgery functional scores (Victorian Institute of Sports Assessment-Achilles questionnaire (VISA-A); 0-100; 100 is perfect function) were taken. For follow-up purpose a questionnaire asking about satisfaction with the result of the operation ('satisfied' or 'not satisfied') and time to return to preinjury sport or recreational activity was used together with the VISA-A score.

\section{RESULTS}

The median follow duration was 10 months (range /7-72 months) after surgery. Before surgery VISA-A scores and questionnaire info were obtained from $9 / 10$ patients (12/13 plantaris tendons). The preoperative VISA-A functional score was 61 (ranging from 45 to 81). After 
surgery, VISA-A scores were obtained from $7 / 10$ patients (8/13 plantaris tendons) and questionnaire information from $7 / 10$ patients. The postoperative VISA-A functional score was 97 (ranging from 94 to 100). All patients who sent back the questionnaire $(7 / 10$ patients) were satisfied and had returned to their preinjury sports or recreational activity. There were no complications in relation to the surgical procedure.

For the patients where scores and questionnaire are missing, we know that one athlete had a quick return to previous activity level, was pain free and competed in Golden League event within 3 months after surgery and continued with pain-free high-level sports for another 2 years, before retiring. Another patient gave information via telephone call and was satisfied and pain free after surgery but retired from high-level sports due to other reasons. From one patient we have no information as he never returned scores or questionnaire, and never answered telephone calls.

\section{DISCUSSION}

Mid-portion Achilles tendinopathy is relatively common among running athletes and recreationally active individuals. However, there are patients complaining of pain on the medial side of the Achilles tendon mid-portion where US and MRI show normal findings. For this subgroup there is often no diagnosis and there has previously been no help to offer. However, with the recent knowledge about the plantaris tendon as a possible source of pain on the medial side of the Achilles tendon there is now a new possible diagnosis.

In this study on a group of elite and recreational athletes suffering from pain on the medial side of the Achilles we found that all had normal Achilles tendons. Using dynamic US examination an often relatively thick plantaris tendon was found to be located close to the Achilles tendon mid-portion in the region for pain during loading and tenderness during palpation. After surgical removal of the plantaris tendon alone, all patients but one (missing data) in this cohort became pain free and returned to their preinjury sport or recreational activity level often within short periods after surgery. These results clearly indicate that the pain was related to the plantaris tendon and the soft tissues surrounding the plantaris. In fact, it has been shown that plantaris tendons exhibit on average more sensory nerves than previously described for the Achilles tendons. ${ }^{5}$ Thus, the plantaris is structurally capable to transmit the pain.

The plantaris tendon is well known to be difficult to localise/identify, and earlier anatomical studies have claimed that up to $20 \%$ individuals lack this tendon. ${ }^{10}$ However, recent research on cadavers (following the plantaris from proximal to distal) has shown that most likely all individuals have a plantaris tendon, but the course and insertion of the plantaris tendon varies, and up to nine different positions in relation to the Achilles mid-portion have been reported. ${ }^{9}$ US has been shown to be useful to identify and follow the course of the plantaris tendon, ${ }^{910}$ but the method does not pick $100 \%$ of the plantaris tendons, showing that due to the positioning of the plantaris tendon in certain individuals it is difficult to separate from the Achilles tendon. This is also our clinical experience, where we in rare cases find the plantaris tendon to be invaginated into the medial side of the Achilles tendon.

The individuals in our study were all involved in sports and recreational activity, and $7 / 10$ were track and field athletes (sprinters, long jump, pool vault), with forceful explosive full-range ankle joint movements in their sport. All complained from having sharp pain on the medial side of the Achilles when pushing off, a type of pain that was so strong that they could not run through it. It appears that loading in maximal plantar flexion might be a risk factor for individuals having a plantaris tendon located close to the medial side of the Achilles. In a recent cadaver study, using US and macroscopical dissection, it was shown that the plantaris tendon had three different movement planes: superior-inferior, anterior-posterior and medial-lateral. ${ }^{8}$ With this information it is likely that depending on the individual positioning of the plantaris tendon, certain ankle joint movements will provoke the plantaris and adjacent tissues differently. Of interest from this patient cohort is that all patients had felt a worsening from trying treatment with eccentric calf muscle training. This indicates that going from loaded maximum plantar flexion to maximal dorsiflexion provokes plantaris tendon-related pain, and if this also is found in larger population studies, it can be used as part of the diagnostic tools for this diagnosis.

This study includes a rather small material, 10 patients with 13 painful plantaris tendons, and larger materials are needed for stronger conclusions to be made. However, our clinical experience is that plantaris tendon-related pain alone is rare. Most patients complaining from pain in the Achilles tendon mid-portion have Achilles tendinopathy alone, and sometimes together with plantaris tendon involvement. The group of patients with plantaris tendon-related pain alone, having a normal Achilles, is small and it will take time to get large materials. Another weakness in this study is that we could not get follow-up details from all patients, and that is unfortunately a common problem when involving athletes, maybe especially when they like in this study come from different countries.

In conclusion, we suggest to keep the plantaris tendon in mind when evaluating painful conditions in the Achilles tendon region. Plantaris tendinopathy-related pain alone and normal Achilles tendon exist. Short-term results from excision of the plantaris tendon via a minor surgical procedure in local anaesthesia have been shown to be good.

Acknowledgements The authors thank all the patients for their willingness to participate in this study.

Contributors HA performed all surgeries and clinical examinations. CS performed the data analysis and wrote main parts of the manuscript. LM has contributed 
considerably to patient recruitment and manuscript writing. All authors were involved in the design of the study, data collection and manuscript writing. The manuscript was finally approved by all authors.

Funding The authors have not declared a specific grant for this research from any funding agency in the public, commercial or not-for-profit sectors.

Competing interests None declared.

Patient consent for publication Not required.

Ethics approval Studies on Achilles and plantaris tendinopathy were approved by the local ethics committee (Umea University, Sweden).

Provenance and peer review Not commissioned; internally peer reviewed.

Open access This is an open access article distributed in accordance with the Creative Commons Attribution Non Commercial (CC BY-NC 4.0) license, which permits others to distribute, remix, adapt, build upon this work non-commercially, and license their derivative works on different terms, provided the original work is properly cited, appropriate credit is given, any changes made indicated, and the use is non-commercial. See: http://creativecommons.org/licenses/by-nc/4.0

\section{REFERENCES}

1. Cook JL, Purdam CR. The challenge of managing tendinopathy in competing athletes. Br J Sports Med 2014;48:506-9.

2. Habets $B$, van Cingel RE. Eccentric exercise training in chronic mid-portion Achilles tendinopathy: a systematic review on different protocols. Scand J Med Sci Sports 2015;25:3-15.

3. Alfredson $\mathrm{H}$. Ultrasound and Doppler-guided mini-surgery to treat midportion Achilles tendinosis: results of a large material and a randomised study comparing two scraping techniques. Br J Sports Med 2011;45:407-10

4. Spang C, Alfredson $\mathrm{H}$, Ferguson $\mathrm{M}$, et al. The plantaris tendon in association with mid-portion Achilles tendinosis: tendinosis-like morphological features and presence of a non-neuronal cholinergic system. Histol Histopathol 2013;28:623-32.
5. Spang C, Harandi VM, Alfredson $\mathrm{H}$, et al. Marked innervation but also signs of nerve degeneration in between the Achilles and plantaris tendons and presence of innervation within the plantaris tendon in midportion Achilles tendinopathy. J Musculoskelet Neuronal Interact 2015;15:197-206.

6. Masci L, Spang C, van Schie HT, et al. How to diagnose plantaris tendon involvement in midportion Achilles tendinopathy - clinical and imaging findings. BMC Musculoskelet Disord 2016;17:97.

7. Alfredson $\mathrm{H}$. Midportion achilles tendinosis and the plantaris tendon. Br J Sports Med 2011;45:1023-5.

8. Smith J, Alfredson H, Masci L, et al. Differential plantaris-achilles tendon motion: a sonographic and cadaveric investigation. $P m R$ 2017:9:691-8.

9. van Sterkenburg MN, Kerkhoffs GM, Kleipool RP, et al. The plantaris tendon and a potential role in mid-portion Achilles tendinopathy: an observational anatomical study. J Anat 2011;218:336-41.

10. Spang C, Alfredson $\mathrm{H}$, Docking SI, et al. The plantaris tendon: a narrative review focusing on anatomical features and clinical importance. Bone Joint J 2016;98-B:1312-9.

11. van Sterkenburg MN, van Dijk CN. Mid-portion achilles tendinopathy: why painful? An evidence-based philosophy. Knee Surg Sports Traumatol Arthrosc 2011;19:1367-75.

12. Cook JL, Purdam C. Is compressive load a factor in the development of tendinopathy? Br J Sports Med 2012;46:163-8.

13. Ruergård $\mathrm{A}$, Alfredson $\mathrm{H}$. Major physical but also psychological effects after pain relief from surgical scraping in patients with Achilles tendinopathy -A 1-year follow-up study. Pain Studies and Treatment 2014;02:21-5.

14. Calder JD, Freeman R, Pollock N. Plantaris excision in the treatment of non-insertional Achilles tendinopathy in elite athletes. Br J Sports Med 2015:49:1532-4.

15. Masci L, Spang C, van Schie HT, et al. Achilles tendinopathy-do plantaris tendon removal and Achilles tendon scraping improve tendon structure? A prospective study using ultrasound tissue characterisation. BMJ Open Sport Exerc Med 2015;1:e000005

16. Bedi HS, Jowett $C$, Ristanis $S$, et al. Plantaris excision and ventral paratendinous scraping for achilles tendinopathy in an athletic population. Foot Ankle Int 2016;37:386-93. 